\title{
Alcohol intake and risk of colorectal cancer: Results from the UK Dietary Cohort Consortium
}

\author{
JY Park', CC Dahm², RH Keogh ${ }^{2,3}$, PN Mitrou², BJ Cairns ${ }^{4}$, DC Greenwood ${ }^{5}$, EA Spencer ${ }^{4}$, IS Fentiman ${ }^{6}$, \\ MJ Shipley, EJ Brunner ${ }^{7}$, JE Cade ${ }^{5}$, VJ Burley ${ }^{5}$, GD Mishra ${ }^{8}$, D Kuh ${ }^{8}$, AM Stephen', IR White ${ }^{3}$, RN Luben', \\ AA Mulligan', K-T Khaw, and SA Rodwell, ${ }^{2}, 10$
}

'Department of Public Health and Primary Care, University of Cambridge, Cambridge CBI 8RN, UK; ${ }^{2}$ Department of Public Health and Primary Care, Medical Research Council Centre for Nutritional Epidemiology in Cancer Prevention and Survival, University of Cambridge, Cambridge CBI 8RN, UK; ${ }^{3}$ Medical Research Council Biostatistics Unit, Institute of Public Health, University of Cambridge, Cambridge CB2 OSR, UK; ${ }^{4}$ Cancer Epidemiology Unit, University of Oxford, Oxford OX3 7LF, UK; ${ }^{5}$ Centre for Epidemiology and Biostatistics, Faculty of Medicine and Health, University of Leeds, Leeds LS2 9JT, UK; ${ }^{6}$ Academic Oncology Unit, Guy's Hospital, London, SEI 9RT, UK; ${ }^{7}$ Department of Epidemiology and Public Health, University College London, London WCIE 6BT, UK; ${ }^{8}$ Department of Epidemiology and Public Health, Medical Research Council Unit for Lifelong Health and Ageing, University College London, 33 Bedford Place, London WCIB 5JU, UK; ${ }^{9}$ Medical Research Council Human Nutrition Research, Elsie Widdowson Laboratory, Cambridge CBI 9NL, UK

BACKGROUND: Epidemiological studies have suggested that excessive alcohol intake increases colorectal cancer (CRC) risk. However, findings regarding tumour subsites and sex differences have been inconsistent.

METHODS: We investigated the prospective associations between alcohol intake on overall and site- and sex-specific CRC risk. Analyses were conducted on 579 CRC cases and 1996 matched controls nested within the UK Dietary Cohort Consortium using standardised data obtained from food diaries as a main nutritional method and repeated using data from food frequency questionnaire (FFQ).

RESULTS: Compared with individuals in the lightest category of drinkers ( $>0-<5 \mathrm{~g}$ per day), the multivariable odds ratios of CRC were I.I 6 (95\% confidence interval $(95 \% \mathrm{Cl})$ : 0.88, I.53) for non-drinkers, 0.9 I $(95 \% \mathrm{Cl}: 0.67, \mathrm{I} .24)$ for drinkers with $5-<15 \mathrm{~g}$ per day, 0.90 (95\% Cl: 0.65, I.25) for drinkers with I $5-<30$ g per day, I.02 (95\% Cl: 0.66, I.58) for drinkers with $30-<45 \mathrm{~g}$ per day and I. 19 (95\% Cl: $0.75,1.9$ I) for drinkers with $\geqslant 45 \mathrm{~g}$ per day. No clear associations were observed between site-specific CRC risk and alcohol intake in either sex. Analyses using FFQ showed similar results.

CONCLUSION: We found no significantly increased risk of CRC up to $30 \mathrm{~g}$ per day of alcohol intake within the UK Dietary Cohort Consortium.

British Journal of Cancer (2010) I 03, 747-756. doi:I0.I038/sj.bjc.6605802 www.bjcancer.com

Published online 20 July 2010

(c) 2010 Cancer Research UK

Keywords: colorectal cancer; alcohol intake; prospective cohort study; food diary; food frequency questionnaire

The descriptive epidemiology of colorectal cancer (CRC) shows significant geographical variation in incidence rates worldwide and provides strong circumstantial evidence that lifestyle has an important role in colorectal carcinogenesis (Stewart and Kleihues, 2003). Alcohol drinking is one such important lifestyle factor (Ferrari et al, 2007): the IARC (International Agency for Research on Cancer) recently added CRC to the list of alcohol-related malignancies (Baan et al, 2007), and the 2007 World Cancer Research Fund/American Institute for Cancer Research Expert Report (WCRF/AICR Report) concluded that intake of ethanol from alcoholic drinks of $>30 \mathrm{~g}$ per day is a convincing cause of CRC in men and a probable cause in women (WCRF/AICR, 2007).

\footnotetext{
*Correspondence: Professor K-T Khaw;

E-mail: kkl0I@medschl.cam.ac.uk

${ }^{10}$ Professor Rodwell (professionally known as Bingham) read an initial draft of this manuscript, but sadly passed away in June 2009.

Received 13 April 2010; revised 9 June 2010; accepted 18 June 2010; published online 20 July 2010
}

In the United Kingdom, $30 \mathrm{~g}$ of alcohol is equivalent to $3-4$ units, 1 unit being $\sim 8 \mathrm{~g}$ of alcohol (The National Health Service, 2010). Associations between alcohol intake and CRC risk according to anatomical subsites of the colorectum remain unclear (Chen et al, 2005; Akhter et al, 2007; Ferrari et al, 2007; Bongaerts et al, 2008; Lim and Park, 2008), although it is believed that colon and rectal cancers have different aetiologies (Li and Lai, 2009), and that within the colon, proximal and distal sites have biologically distinct functions (Bufill, 1990; Lindblom, 2001). Evidence has mostly been available for men with high alcohol intake (Allen $e t$ al, 2009), and risks of CRC with alcohol intake for men and women have not been consistent.

Many epidemiological studies which investigated an effect of alcohol on health have relied on self-reports of alcohol intakes. Owing to its simplicity in use and convenience in administration, food frequency questionnaires (FFQs) have been mostly used in alcohol intake assessment (Feunekes et al, 1999). However, as a nutritional instrument, FFQs may have both large random and systematic measurement errors (Bingham et al, 2003; Prentice, 2003; Schatzkin et al, 2003), and for a number of nutrients, food 
diaries have been shown to provide measurements that are more strongly associated with biomarker data (Bingham et al, 1997, 2008; Day et al, 2001). Furthermore, it has been suggested that food diaries can capture a more complicated individual dietary intake more accurately (Bingham et al, 2003). However, less is known about whether food diaries provide a superior measure of food intake for infrequently or episodically consumed items, such as alcoholic drinks, compared with the FFQs. Therefore, it is important to compare the effects of alcohol intake on CRC risk using food diaries and FFQs.

In the United Kingdom, government recommendations on alcohol intake are for men to consume no more than 3-4 units per day ( $<32 \mathrm{~g}$ per day) and for women to consume no more than $2-3$ units per day ( $<24 \mathrm{~g}$ per day) (The National Health Service, 2010); however, the average annual alcohol intake in the United Kingdom now exceeds the European Union average (Department of Health, 2009) and CRC is the second major cause of cancer death in the country (Westlake and Cooper, 2008). Worldwide, more than one million incident cases were recorded in 2002 (WCRF/AICR, 2007). Hence, even a moderate association between alcohol intake and CRC risk may have important public health implications.

The aim of this study was to examine the relationship between alcohol intake and overall and site-specific CRC risks, including differences in sex-specific risks, using a case-control study nested within the UK Dietary Cohort Consortium, from which nutritional data were ascertained by food diaries and FFQs at baseline.

\section{MATERIALS AND METHODS}

\section{Study population}

The UK Dietary Cohort Consortium comprises seven established UK cohorts (namely EPIC-Norfolk, EPIC-Oxford, Guernsey Study, Oxford Vegetarian Study, MRC National Survey of Health and Development (NSHD), the UK Women's Cohort Study (UKWCS) and Whitehall II; Table 1) with a total cohort size of 153000 individuals. The methods of recruitment, study design and ethical approval have been described for each of these cohorts in detail elsewhere (Appleby et al, 1999; Day et al, 1999; Davey et al, 2003; Cade et al, 2004; Allen et al, 2005; Marmot and Brunner, 2005; Wadsworth et al, 2006).

\section{Case ascertainment}

Case patients were individuals who were free of cancer (except non-melanoma skin cancer) at the date of food diary commencement and who developed CRC at least 12 months after the date of diary commencement and before the end of the study period, defined for each study centre by the latest date of complete follow-up for both cancer incidence and vital status.

The last dates of follow-up varied between cohorts, from 31 December 2003 to 1 January 2007. Individuals with selfreported or registry-reported prevalent cancer (except nonmelanoma skin cancer) were omitted from the study. Incident CRC cases (International Statistical Classification of Diseases and Related Health Problems (ICD) 10th Revision, C18-20) were ascertained by record linkage with local cancer registries and the United Kingdom Office for National Statistics, which provided notification of all cancer registrations and deaths by cause for the cohort. For this study, CRC cases were classified according to anatomical subsites: colon cancers were defined as tumours in the caecum, appendix, ascending colon and hepatic flexure, transverse colon, splenic flexure (proximal, C18.0-18.5; ICD 10th Revision), and descending and sigmoid colon (distal, C18.6-C18.7), as well as tumours that were overlapping or unspecified (C18.8 and C18.9). Cancer of the rectum included tumours occurring at the rectosigmoid junction (C19) and rectum (C20). Overall CRC was defined as a combination of all colon and rectal cancer cases.

\section{Selection of matched controls}

Cases were matched within their respective cohort to four controls each, with the exception of some cases from EPIC-Oxford, the Guernsey Study and the Oxford Vegetarian Study who were matched to two controls, and some from the UKWCS who were matched to five controls. Matched controls were selected at random from the appropriate stratum of the set of all cohort members who were free of CRC at the end of follow-up (due to death or censoring) and free of all cancer (except non-melanoma skin cancer) at the date of diary commencement. Matching criteria were sex, age at enrolment ( \pm 3 years) and month of diary completion ( \pm 3 months). Follow-up time for matched controls was also required to be at least as long as that for the case, with follow-up time defined as the time from the date of diary commencement to the date of CRC diagnosis for cases and the time from date of diary commencement until the end of follow-up

Table I Description of studies participating in the UK Dietary Cohort Consortium and summary of alcohol intake among colorectal cancer cases and matched controls ${ }^{\mathrm{a}}$

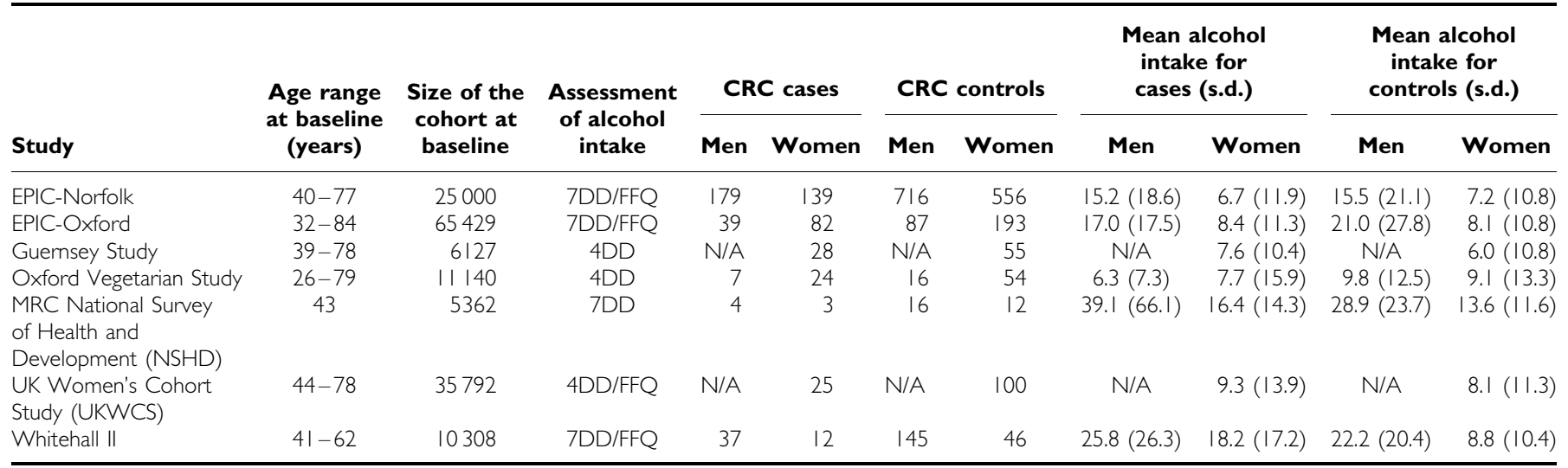

Abbreviations: $\mathrm{CRC}=$ colorectal cancer; $\mathrm{EPIC}=$ European Prospective Investigation into Cancer and Nutrition; MRC = Medical Research Council; 7DD =7-day food diary; $4 \mathrm{DD}=4$-day food diary; $\mathrm{FFQ}=$ food frequency questionnaire; $N / A=$ not applicable. ${ }^{\mathrm{a}} \mathrm{A} t$-test indicated that there was no statistically significant difference in mean alcohol intake between cases and controls. 
for controls. A total of 579 CRC cases and 1996 matched controls were available for analysis.

\section{Diet and lifestyle assessment}

Each cohort collected dietary information using 4-day (Guernsey, Oxford Vegetarian Study, UKWCS) (Appleby et al, 1999; Cade et al, 2004) or 7-day food diaries (EPIC-Norfolk, NSHD, EPIC-Oxford, Whitehall II) (Bingham et al, 2001; Brunner et al, 2001; Davey et al, 2003; Wadsworth et al, 2006) completed on consecutive days at recruitment to the study or during a subsequent monitoring phase. Participants were asked to record in detail all the foods and beverages they consumed, prompted by time slots such as 'Mid-morning - between breakfast time and lunchtime' and also by photographs of standard plates with three different portion sizes of representative foods to help participants estimate the amounts they consumed (Bingham et al, 2001). Information on age, sex, height, weight, smoking status, educational level, social class, physical activity and family history of CRC, were collected either by trained researchers or in questionnaires administered before the completion of the food diary. In four of the seven studies (namely EPIC-Norfolk, EPIC-Oxford, UKWCS and Whitehall II), FFQs were also administered before this data collection, and were available for analysis from most participants in these cohorts. The FFQs were based on that used in the US Nurses' Health Study, listed from 127 to 217 items, and have been validated for use in the United Kingdom (Bingham et al, 1997; Brunner et al, 2001; Cade et al, 2004).

The majority of data from the food diaries were coded to give nutrient intakes and food group information using data entry program Data Into Nutrients for Epidemiological Research (DINER) developed in the EPIC-Norfolk cohort (Welch et al, 2001). A total of 107 UKWCS food diaries were coded and processed using the Diet and Nutrition Tool for Evaluation (DANTE) program (Cade $e t a l$, 2006). We compared 100 food diaries coded under both systems and found good agreement between DANTE and DINER for most nutrients, although the geometric mean intake of alcohol from DINER was $7 \%$ higher (95\% confidence interval (95\% CI): $3-11 \%$ ) than from DANTE.

\section{Alcohol intake assessment}

For the food diaries completed by all centres, beer (stout, bitter, lager; keg, draught, bottled, canned; low alcohol, strong, home-made; number of pints, bottles, cans), cider (sweet, dry, vintage, low alcohol; number of pints, bottles, cans), spirits (what sort: e.g., whisky, gin, vodka, rum; at home or in a pub; single measures as in pub), wine, sherry, port (white, red; sweet, medium, dry; low alcohol; glasses) were assessed for alcohol intake.

The FFQs from EPIC-Norfolk, EPIC-Oxford, UKWCS and Whitehall II were designed to measure a participant's usual food intake during the previous year. In the four centres, FFQs asked participants to estimate how often they drink the following the beverages, 'Beer, larger or cider (half pint)', 'Port, sherry, vermouth, liqueurs (glass)' and 'Spirits, e.g. gin, brandy, whisky, vodka (single)'. For each item on the list, participants were asked to indicate their usual intake, choosing from nine frequency categories, ranging from 'never or less than once per month' to 'more than 6 times per day'.

\section{Statistical analysis}

Conditional logistic regression models were used to estimate odds ratios (ORs) and 95\% CIs for the CRC risk according to alcohol intake, with adjustment for potential confounding variables.

The participants were categorised into six groups according to their baseline alcohol intake, with the lightest category of drinkers
( $>0-<5 \mathrm{~g}$ per day) as a reference group: 0 (non-drinkers), $>0-<5,5-<15,15-<30,30-<45, \geqslant 45 \mathrm{~g}$ per day. An initial unadjusted model was first created to estimate ORs for CRC across categories of alcohol intake. As the matching of cases and controls by age was not exact, the conditional logistic regression models were adjusted for age in years to control for any residual confounding. Multivariable model 1 also adjusted for intakes of energy ( $k$ cal per day), folate ( $\mu \mathrm{g}$ per day), dietary fibre ( $g$ per day), red meat ( $\mathrm{g}$ per day), and processed meat ( $\mathrm{g}$ per day) in addition to height $(\mathrm{m})$, weight $(\mathrm{kg})$, smoking status (never, former, current) and social class (six categories). There were some missing data within studies, with $\sim 1 \%$ of individuals missing weight, height and smoking status, and $\sim 5 \%$ missing social class, all of which were recorded in all studies. The distribution of alcohol intake among individuals with and without these missing data was similar. For these variables, missing values were assumed to be missing at random and were imputed using multiple imputation. In all, 10 imputed data sets were created and multivariable models were fitted using the 'ice' (Royston, 2005) and 'mim' (Carlin, 2008) packages in STATA (Stata Corporation, College Station, TX, USA). Multivariable model 2 adjusted for physical activity (inactive, moderately inactive, moderately active and active) and educational level (none, GCSE (completed to age 15 years), A Level (completed to age 17 years) and degree level) in addition to the adjustments in multivariable model 1. Data on physical activity level were not available for NSHD and the Guernsey Study, and information on educational level was not available for the Oxford Vegetarian Study. The effects of adjustment for these variables were assessed by fitting multivariable models 1 and 2 using the subset of participants (458 cases and 1734 controls) with complete information on physical activity and educational level. Sex-specific and anatomical subsite-specific models were also fitted using multivariable models 1 (579 cases and 1996 controls) and 2 (458 cases and 1734 controls). Tumours that were overlapping or unspecified were not included in site-specific analyses of the proximal and the distal colon cancer $(n=60)$.

To investigate whether different nutritional instruments might alter our results, we repeated the analyses using FFQ data. Dietary data obtained from FFQs were available for participants in EPICNorfolk, EPIC-Oxford, the UKWCS and Whitehall II (496 cases and 1809 controls). These analyses were restricted to those 2305 participants who completed both the FFQ and the food diary, and ORs were estimated using multivariable models 1 and 2 .

Tests for trend were conducted by modelling alcohol intake as a continuous variable in a conditional logistic regression analysis. To assess the possibility of a non-linear association between alcohol intake and CRC risk, the multivariable models were fitted with the inclusion of a quadratic term for continuous alcohol intake. Simple associations between categorical covariates and alcohol intake were assessed using Pearson's $\chi^{2}$ tests for two independent proportions. For continuous variables, means across categories of alcohol intake were compared by $t$-tests, analysis of variance or a Kruskal-Wallis test (for red meat and processed meat intake only). All statistical tests were two sided, and all statistical analyses were performed using the statistical software package STATA (version 10).

\section{RESULTS}

A total of 579 incident CRC cases and 1996 matched controls were available for analysis from the 7 participating UK cohorts. Of these cancer cases, 380 were located in the colon and 199 in the rectum. There were no statistically significant differences in the means of alcohol intake between cases and controls in each cohort (Table 1).

Table 2 presents participant characteristics according to categories of alcohol intake. Among drinkers, $82 \%$ consumed $<30 \mathrm{~g}$ per day alcohol. The average alcohol intake was $\sim 17 \mathrm{~g}$ per day 
Table 2 Distribution of participant characteristics by categories of alcohol intake as assessed by food diaries, shown separately for men and women ${ }^{\text {a }}$

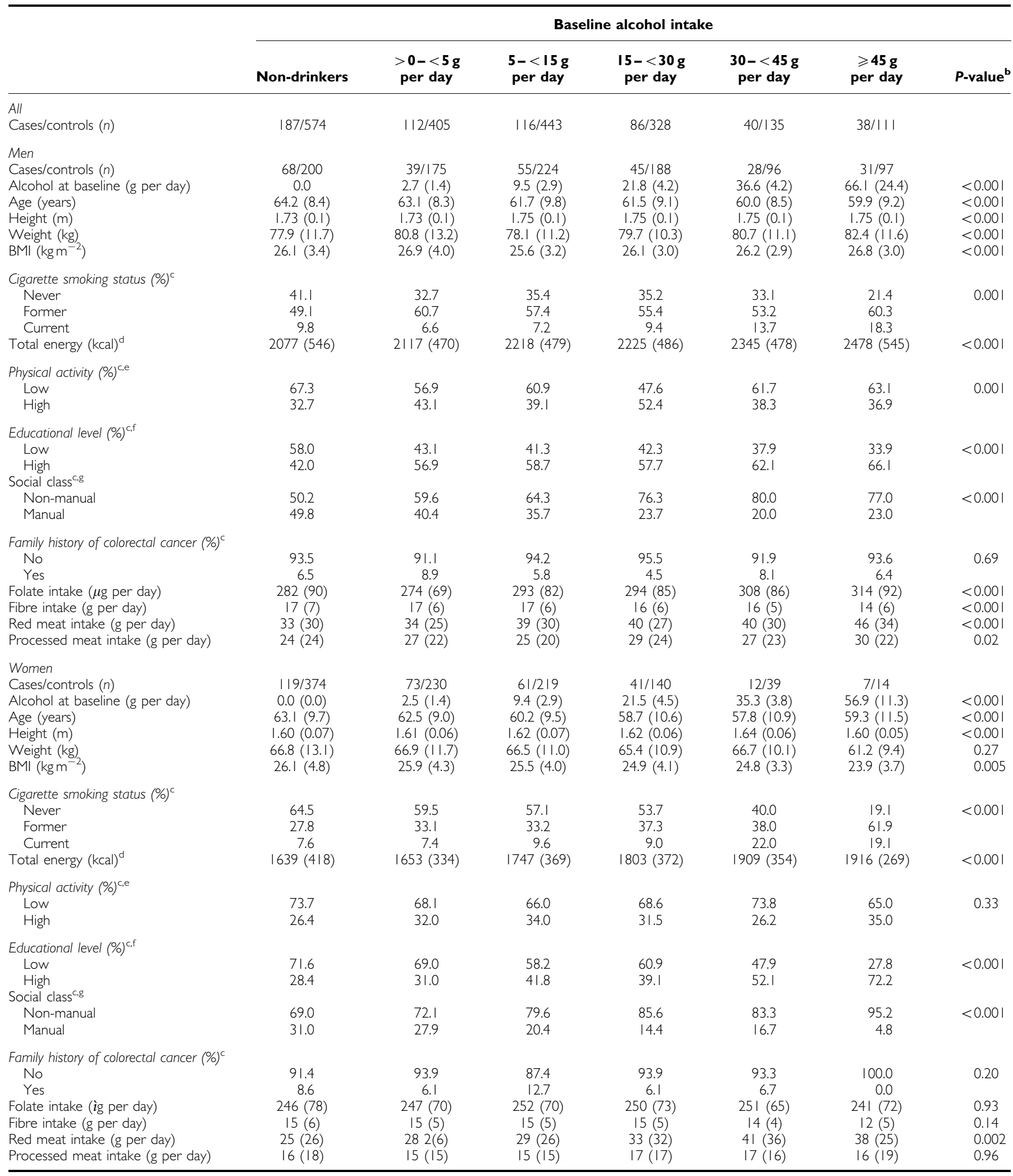

Abbreviation: BMI = body mass index. ${ }^{\mathrm{a}}$ Mean (s.d.) or number (\%), and P-values for tests of association. ${ }^{\mathrm{b}}$ For continuous variables, analysis of variance or a Kruskal-Wallis test (for red meat and processed meat intake) was used to test whether the variables differed significantly across categories of alcohol intake. For categorical variables, $\chi^{2}$ tests

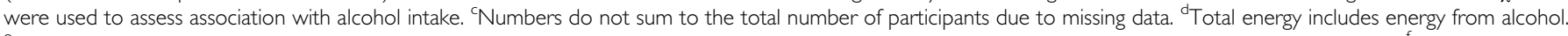
eLow physical activity was defined as being inactive or moderately inactive, and high physical activity was defined as being moderately active or active. ${ }^{\mathrm{f} E d u c a t i o n a l}$ levels were regrouped into low educational level (no qualification or General Certificate of Secondary Education (GCSE) level or equivalent) and high educational level (degree or equivalent, A-level or equivalent). ${ }^{8}$ Social class was classified according to the Registrar General's occupation-based classification scheme and was dichotomised into non-manual (social class I, II and IIInm) and manual (IIIm, IV and V). 
Table 3 Odds ratios (95\% confidence intervals) from multivariable models for colorectal cancer risk in categories of total alcohol intake as assessed by food diaries

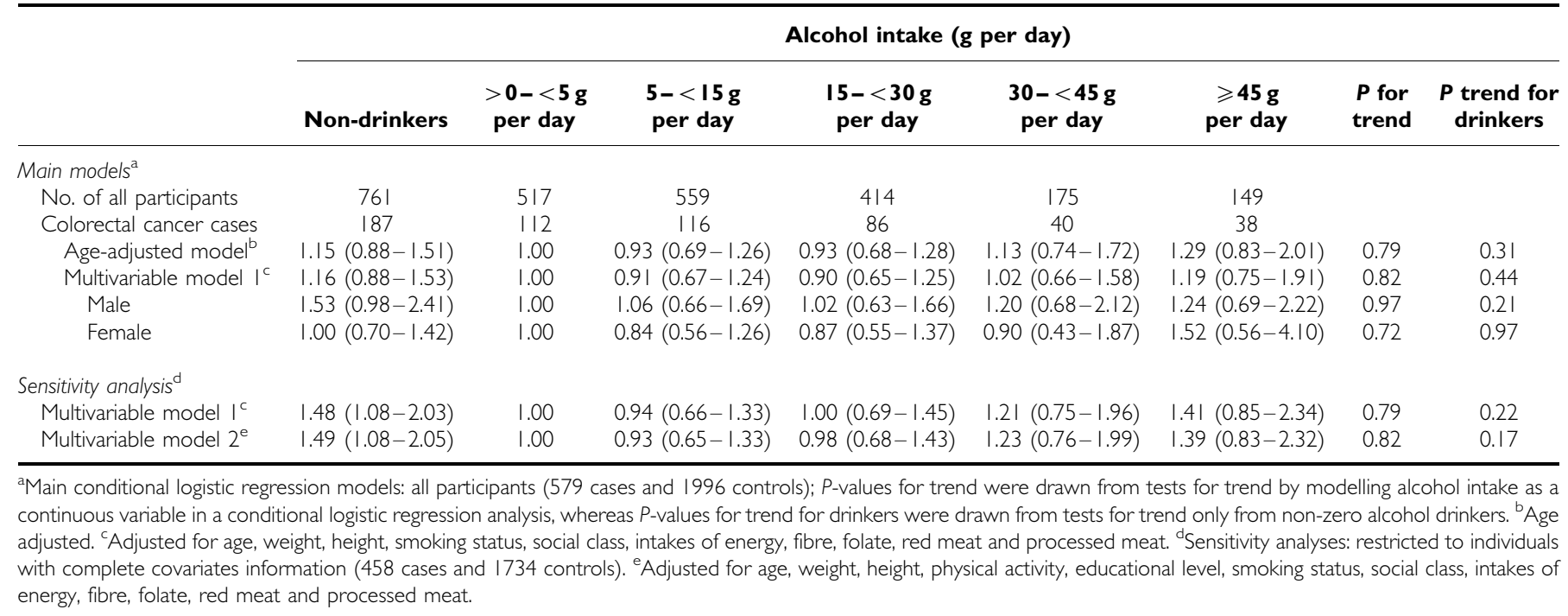

( $\sim 2$ units per day) for men and $8 \mathrm{~g}$ per day (1 unit per day) for women. Men less frequently reported being non-drinkers and more frequently reported drinking $\geqslant 30 \mathrm{~g}$ per day of alcohol than did women. Men who consumed $\geqslant 30 \mathrm{~g}$ per day were significantly younger and had slightly higher BMI compared with those who consumed $<30 \mathrm{~g}$ per day. These men with $\geqslant 30 \mathrm{~g}$ per day of alcohol intake more frequently reported being former or current smokers, had higher energy intake, were physically less active and had attained a higher educational level, as well as being more likely to be non-manual workers. Similar patterns were seen among women, although women with $\geqslant 30 \mathrm{~g}$ per day of alcohol intake had a lower mean BMI compared with non-drinkers, and there was no significant difference in physical activity levels across categories of alcohol intake.

Table 3 shows the ORs for CRC by categories of alcohol intake as assessed by the food diary from age-adjusted and multivariable models. Non-drinkers had a moderate, non-significant increased risk compared with those who drank $>0-<5$ g per day $(<1$ units per day) in the main models. As we were unable to differentiate individuals who did not drink in the time period during which their food diaries were recorded from never drinkers (former drinkers or life-long never drinkers), the category of non-drinkers might include temporary non-drinkers who are in fact drinkers. Therefore, we focused on analyses from individuals who reported non-zero alcohol intake.

In general, alcohol intake was not significantly associated with the risk of CRC (Table 3). Compared with individuals in the lowest category of alcohol intake among drinkers $(>0-<5 \mathrm{~g}$ per day), individuals in the highest category of intake who consumed $\geqslant 45$ g per day ( $\sim 6$ units per day) did not have a significantly higher CRC risk before or after adjustment for age, weight, height, smoking status, social class and intakes of energy, fibre, folate, red meat and processed meat (OR: 1.19; 95\% CI: 0.75, 1.91). None of the other categories showed a significant association with CRC risk compared with the group consuming $>0-<5 \mathrm{~g}$ per day of intake. No significant sex-specific associations were observed between alcohol intake and CRC risk. When we conducted further analyses adjusting for non-alcohol energy and the same covariates used in multivariable models, the results scarcely differed and they did not vary by sex.

In the sensitivity analysis in which further adjustment for physical activity and educational level was made in a subset of the study population with complete covariate information, being a non-drinker was significantly associated with an increased CRC risk. However, this result was seen under both multivariable models 1 and 2 in the sensitivity analysis, indicating that the result is not due to adjustment for physical activity and educational level but rather to the omission of some cohorts from the analysis. The point estimates for the highest category of alcohol intake tended to be higher in this subset of studies (OR: 1.41; 95\% CI: 0.85, 2.34 for $\geqslant 45 \mathrm{~g}$ per day). As in the main analyses, adjustment for physical activity and educational level did not alter the results in the subset.

Multivariable models 1 and 2 were suggestive of a J-shaped association between alcohol intake and CRC risk. However, a further analysis using continuous alcohol intake with a quadratic term provided no evidence for a non-linear association between alcohol intake and CRC risk ( $P$ for quadratic term $=0.17$ for drinkers). Additional adjustment for family history of CRC (343 cases and 1370 controls) did not make substantial differences to ORs (data not shown).

When we investigated these associations further by tumour subsites (stratified by sex), no clear associations were observed between risks of overall colorectum, proximal/distal colon, or rectum and alcohol intake in both sexes (Table 4). The analysis using multivariable model 2 for the subset of individuals with information on physical activity and educational level showed increased distal colon cancer risk for alcohol intake of $\geqslant 30 \mathrm{~g}$ per day compared with intake of $>0-<5 \mathrm{~g}$ per day (OR: 2.36 ; $95 \%$ CI: $1.13,4.91, P$ trend for drinkers $=0.03$ ). However, this may be a chance finding.

Using data obtained from food diaries, we were also able to examine the association between specific alcoholic beverage intake and risk of CRC. When we calculated multivariable ORs per 1 s.d. increase in intake of beer $(280 \mathrm{~g})$, wine $(70 \mathrm{~g})$, spirits $(20 \mathrm{~g})$ and fortified wine $(24 \mathrm{~g})$, no clear associations were observed. The results did not vary by sex (data not shown).

Table 5 and Figure 1 show a comparison of the results from using FFQ and food diary to obtain measurements of alcohol intake. Analyses using FFQ resulted in a similar pattern of associations to those using food diaries. The association between alcohol intake and CRC risk remains statistically non-significant using FFQ, although suggests an increasing trend in the OR estimates with increasing alcohol intake $(P$ for trend $=0.09$ among drinkers in multivariable model 1). The distribution of participants across the categories of alcohol intake differed in the FFQ and food diary data. Among the subset of participants with both measurements $(n=2305)$, out of 646 individuals who reported zero intake on the food diary, 305 (47\%) reported being 


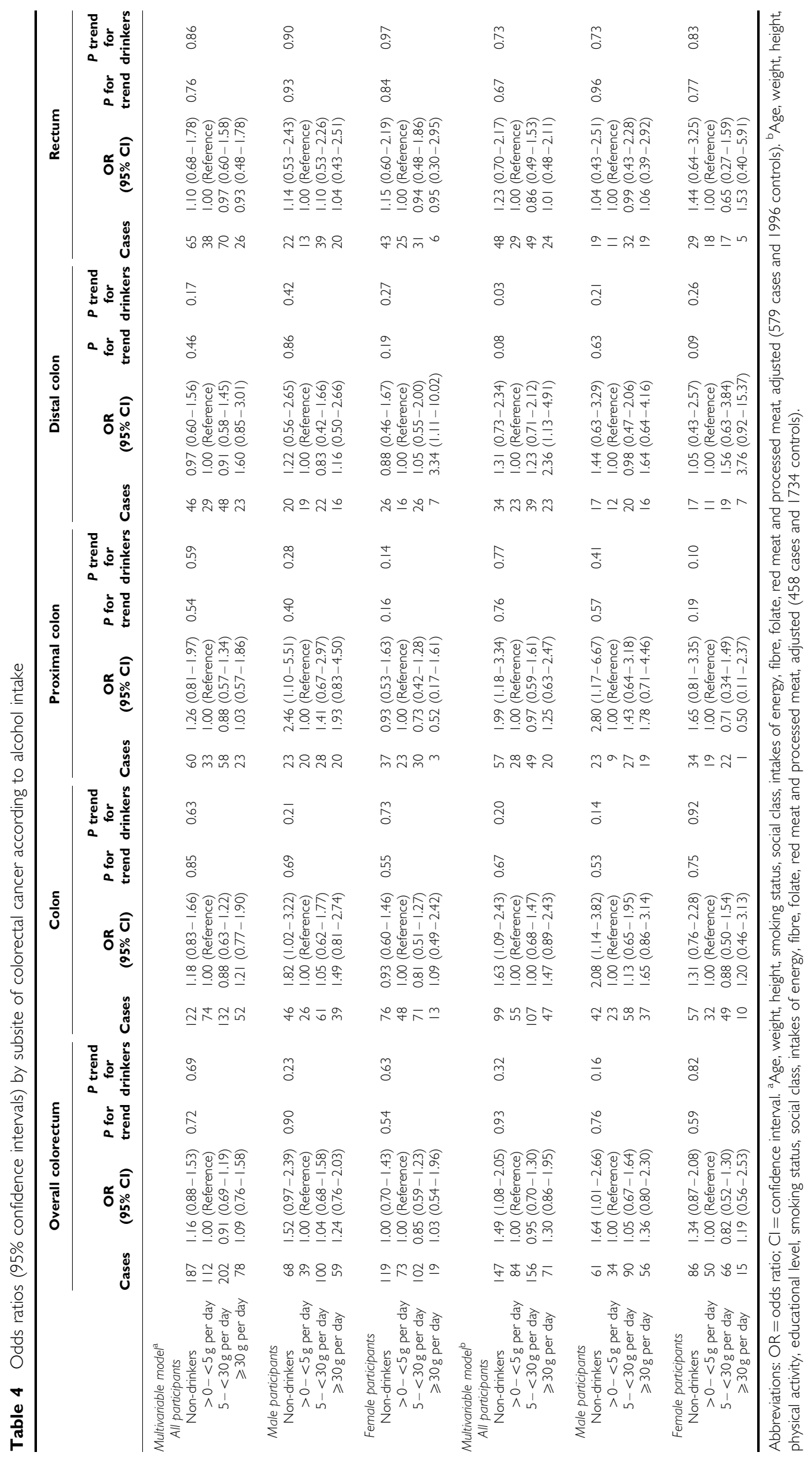


Table 5 Odds ratios (95\% confidence intervals) from multivariable models for colorectal cancer risk in categories of alcohol intake as assessed by food diaries and FFQs among participants with both measures ${ }^{a}$

\begin{tabular}{|c|c|c|c|c|c|c|c|c|}
\hline & \multicolumn{8}{|c|}{ Alcohol intake (g per day) } \\
\hline \multicolumn{9}{|l|}{ Food diaries } \\
\hline No. of all participants & 646 & 477 & 510 & 371 & 165 & 136 & & \\
\hline Colorectal cancer cases & 149 & 100 & 100 & 75 & 38 & 34 & & \\
\hline Multivariable model $\mathrm{I}^{\mathrm{b}}$ & $1.18(0.88-1.60)$ & 1.00 & $0.91(0.66-1.26)$ & $0.92(0.65-1.30)$ & $1.08(0.68-1.70)$ & $1.24(0.76-2.04)$ & 0.97 & 0.60 \\
\hline \multicolumn{9}{|l|}{ FFQs } \\
\hline No. of all participants & & 867 & 662 & 226 & 100 & 78 & & \\
\hline Colorectal cancer cases & 84 & 171 & 150 & 46 & 26 & 19 & & \\
\hline Multivariable model $\mathrm{I}^{\mathrm{b}}$ & $1.43(1.04-1.97)$ & 1.00 & $1.22(0.94-1.58)$ & $1.16(0.79-1.72)$ & $1.36(0.81-2.28)$ & $1.40(0.79-2.49)$ & 0.12 & 0.09 \\
\hline Multivariable model $2^{c}$ & $1.33(0.96-1.86)$ & 1.00 & $1.16(0.87-1.53)$ & $1.07(0.7|-| .6 \mid)$ & $1.18(0.68-2.03)$ & $1.30(0.72-2.38)$ & 0.36 & 0.07 \\
\hline
\end{tabular}

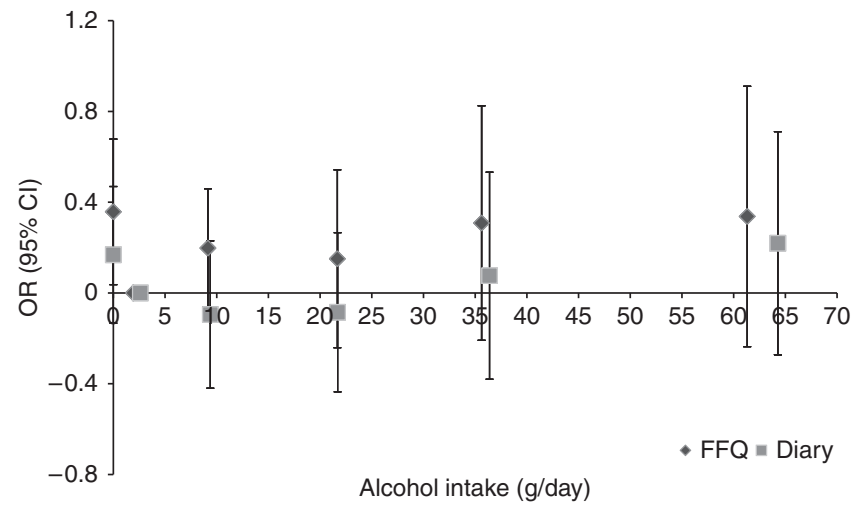

Figure I Comparison of odds ratios (ORs) in a log scale for categories for alcohol intake data $(0,>0-<5$ (reference), $5-<15,15-<30,30-$ $<45$ and $\geqslant 45$ g per day) obtained by food diaries or FFQs. A total of 2305 study participants had complete alcohol intake information from both diaries and FFQs ( $n=496$ cases and 1809 controls). Ors for each category were plotted against the mean alcohol intake (g per day) for each category $(0,2.6,9.4,21.7,36.4$ and $64 \mathrm{~g}$ per day for food diaries and 0, I.9, 9. I, 21.7, 35.6 and $61.3 \mathrm{~g}$ per day for FFQs, respectively) and were adjusted for age, weight, height, smoking status, social class, intakes of fibre and folate.

non-drinkers on the FFQ. Approximately $95 \%$ of individuals $(n=613)$ reporting zero alcohol intake on the food diary consumed $<5$ g per day of alcohol according to the FFQ. A total of 67 individuals $(18 \%)$ reported zero alcohol intake on FFQ and $>0$ alcohol intake on the food diary.

Sex-specific analyses of the linear association between CRC risk and an increase in alcohol intake of $8 \mathrm{~g}$ per day ( 1 unit per day) showed no clear linear associations in either sex (OR: $0.99 ; 95 \%$ CI: $0.93,1.05$ for men, OR: 1.01 ; 95\% CI: $0.92,1.12$ for women in multivariable model 1). The results scarcely differed from the analyses using drinkers only. When we examined interactions between alcohol intake and BMI $\left(<25 \mathrm{~kg} \mathrm{~m}^{-2}, \geqslant 25 \mathrm{~kg} \mathrm{~m}^{-2}\right)$, smoking status (never, former or current), the $P$-values for the interaction were 0.26 for BMI and 0.53 for smoking status. The Reference Nutrient Intake in the United Kingdom for folate is $200 \mu \mathrm{g}$ per day (Department of Health, 1999). When folate intake was dichotomised below and above this level, the $P$-value for interaction was 0.59 . There was no evidence of heterogeneity between centres in the association between alcohol intake and CRC risk in the different centres $(P=0.30)$. Centre-specific ORs for CRC per $8 \mathrm{~g}$ per day of alcohol intake (1 unit per day) were computed (Supplementary Data). The summary OR estimate for $8 \mathrm{~g}$ per day increase in alcohol intake was derived by fixed effects meta-analysis and found to be 1.00 (95\% CI: $0.95,1.05)$ after adjusting for age, and intakes of energy, folate, fibre and red and processed meat.

\section{DISCUSSION}

In this large nested case-control study of 579 CRC cases and 1996 matched controls, alcohol intake within the observed range was not associated with a significantly increased CRC risk after multivariable adjustment when compared with alcohol intake of $>0-<5 \mathrm{~g}$ per day. In subgroup analyses of cancer sites including proximal/distal colon and rectum, no clear associations were observed with total alcohol intake. There was also no evidence of a difference between men and women in the association between alcohol intake and CRC risk. Analyses using a subset of participants who had completed both FFQs and food diaries showed similar shaped associations using each of the two instruments, although risk estimates were higher but still statistically nonsignificant when using FFQ data.

A meta-analysis of prospective cohort studies showed $19 \%$ of increased risk of CRC with an increase of $100 \mathrm{~g}$ per week in alcohol intake (Moskal et al, 2006). Recent cohort studies in which FFQs were the main nutritional instrument have shown no association (Chen et al, 2005), or a significant adverse effect of alcohol when intake is greater than $\sim 16 \mathrm{~g}$ per day (Toriola et al, 2008), $30 \mathrm{~g}$ per day (Ferrari et al, 2007; Bongaerts et al, 2008; Mizoue et al, 2008) or $\sim 45 \mathrm{~g}$ per day (Akhter et al, 2007) compared with study-specific reference groups of lower intakes. These individual studies have not found consistent results in sex- and subsite-specific analyses, with several studies finding greater risk of rectal than colon cancer for alcohol intake of $\geqslant 30 \mathrm{~g}$ per day (Ferrari et al, 2007; Bongaerts et al, 2008). The Million Women Study recently reported a positive association between moderate alcohol intake ( $>15$ drinks per week) and rectal cancer risk but found no evidence of increased colon cancer risk among middle-aged women (Allen et al, 2009). Previous 
studies have, however, failed to reach clear consensus on the association between moderate alcohol drinking ( $<30 \mathrm{~g}$ per day) and colon or rectal cancer risk, and there are still few studies which have investigated proximal and distal colon cancer separately.

It has been suggested that the aetiology of CRC varies by subsite (Stang and Kluttig, 2008; Li and Lai, 2009). The proximal and distal colons have different embryonic origins and their physiology and functions may vary (Stang and Kluttig, 2008). Studies have also shown that microsatellite instability is often linked to proximal colon cancer, whereas chromosomal instability is more common in distal colon cancer (Lindblom, 2001). Therefore, subsite-specific studies are required for a better understanding of the aetiology of CRC. Our study, exploring CRC subsites in men and women in detail, suggested elevated risk of distal colon cancer, for individuals with alcohol intake of $\geqslant 30 \mathrm{~g}$ per day compared with $>0-<5$ g per day and a possible dose-response relationship among drinkers when analysed for the subset of cohorts with complete covariate information. Thus, future studies are warranted focusing on a possible role of alcohol use in the risk of colon cancer, especially proximal or distal colon cancer.

The Panel of the WCRF/AICR Report judged that the evidence of alcohol intake of $>30 \mathrm{~g}$ per day as a cause of CRC is convincing in men and probable in women (WCRF/AICR, 2007), based on a sex-specific meta-analysis finding summary effect estimates of 1.09 (95\% CI: $1.02,1.15)$ per $10 \mathrm{~g}$ per day increase in alcohol intake for men, based on 7 cohort studies, and 1.00 (95\% CI: 0.89, 1.40) for women, based on 3 cohort studies. There were no statistically significant differences in association by cancer site. The threshold of $30 \mathrm{~g}$ per day of alcohol intake is from the results of the pooled analysis of eight cohort studies in which no increased risk was observed below the threshold (Cho et al, 2004).

Our results are consistent with the 2007 WCRF/AICR Report. We found no increased risk of CRC up to $30 \mathrm{~g}$ per day of alcohol intake, with no substantial differences detected in subsite-specific analyses. Although men and women have been shown to have different physiological responses to alcohol (Ely et al, 1999) and the effect of alcohol in our study seemed larger in men (OR: 1.24, 95\% CI: 0.76-2.03 for drinkers with $\geqslant 30 \mathrm{~g}$ per day compared with the lightest category drinkers $(>0-<5 \mathrm{~g}$ per day)) than in women (OR: $1.03,95 \%$ CI: $0.54-1.96$ for drinkers with $\geqslant 30 \mathrm{~g}$ per day compared with the lightest category of drinkers $(>0-<5 \mathrm{~g}$ per day)), the associations were not statistically significant. We did not find differential associations with CRC risk by type of alcoholic beverage. This is consistent with the Report which judged that the causal factor is evidently alcohol itself, irrespective of the type of alcoholic drink. A limited number of studies were included in the metaanalysis of alcohol intake and CRC risk in the WCRF/AICR Report. Therefore, our findings contribute to update the current evidence for a future review, confirming no significantly increased risk of CRC with $<30$ g per day of alcohol intake.

The mechanism by which alcohol may influence CRC risk is not well understood (Stewart and Kleihues, 2003). Hypotheses include a local solvent action which facilitates absorption of other carcinogens, for example, a synergetic effect with tobacco smoking (Boffetta and Hashibe, 2006), and an indirect effect through associated deficiencies in nutrients, especially through changes in folate metabolism (Giovannucci et al, 1995). However, in our study, no significant interactions were observed between alcohol intake and folate intake or tobacco smoking with regard to CRC risk.

Our study has several strengths. Its prospective study design precluded bias attributable to differential recall of intake of alcohol by case status. We were able to examine the influence of alcohol intake on site- and sex-specific CRC risk. Furthermore, different types of alcoholic beverages from food diaries were assessed in association with CRC risk.

This study provided the measure of alcohol intake by using both food diaries and FFQs, whereas previous studies on alcohol and CRC risk have relied only on FFQs. The use of food diaries and FFQs for habitually consumed food items have been discussed (Bingham et al, 1997, 2003, 2008). However, there have been few direct attempts to compare those two different nutritional instruments prospectively for episodically consumed food items, including alcohol. Previous studies have shown that FFQs were not inferior in measuring alcohol intake relative to prospective food diaries (Feunekes et al, 1999), and FFQs showed a high level of reproducibility and validity compared with diet records as a reference method (Ferraroni et al, 1996). Our study, which has information both from food diaries and FFQs, found that although FFQs and food diaries cover different durations and measurements may differ between the two instruments, using well-constructed food diaries for measurement of infrequently consumed food items can provide results that do not differ substantially from those using FFQs.

This study used original data from seven UK mature cohorts with standardised diary data entry, which enabled us to create identical categories for alcohol intake across studies that were in line with previous studies (Cho et al, 2004), removing some potential sources of heterogeneity across studies. Furthermore, we were able to adjust for a range of known confounding factors.

An important limitation of this study is that we were unable to differentiate life-long abstainers and former drinkers in the category of non-drinkers in either FFQs or diaries. As previously discussed, many non-drinkers may be former drinkers who had given up drinking because of incipient disease (Doll et al, 1994), although a sensitivity analysis excluding a further 111 cases incident within 3 years of diary completion did not materially change our results. Moreover, in the 4-7-day diaries, we were unable to differentiate non-drinkers from episodic drinkers who happened not to consume alcohol during the time period covered by the diary. Hence, it is likely that the 'non-drinker' category in our diary analyses contains participants who were actually drinkers at the time when diaries were administered. In light of this, we focused on analyses from non-zero alcohol drinkers and reported trend tests for drinkers separately (Allen et al, 2009). We found a moderate positive but non-significant CRC risk in those consuming $\geqslant 30 \mathrm{~g}$ per day of alcohol using data from both food diaries and FFQs. However, in our study, almost half of the participants reported drinking $<5 \mathrm{~g}$ per day in both food diaries and FFQs and only $19 \%$ of men and $17 \%$ of women reported intake in excess of the recommended daily maxima of $3-4$ units $(<32 \mathrm{~g})$ daily for men and $2-3$ units $(<24 \mathrm{~g})$ daily for women. Insufficient participants in the heavier categories prevented us from estimating any potential effect of high alcohol intake with sufficient precision.

Another limitation was that alcohol intake was assessed only once by self-report. As heavy alcohol drinking is considered to be unhealthy, it is likely that individuals underreport their alcohol intake, particularly in the case of heavy intake (Rehm et al, 1999), resulting in overestimation of the actual carcinogenic effect of the habit. In addition, drinking habits are liable to change throughout the lifetime. However, we conducted a sensitivity analysis using data from the EPIC-Norfolk cohort in which information on alcohol intake from participants recalling their habits at ages 20 and 30 years is available, and we again did not find any evidence of an association with CRC risk, although participants tended to report higher alcohol intake at younger ages (data not shown). Nonetheless, more research with additional information on alcohol intake over a longer period of time and on specific drinking behaviour such as binge drinking is required to clarify any hazardous effect of excessive alcohol drinking on CRC risk.

In summary, we found no increased risk of CRC up to $30 \mathrm{~g}$ per day of alcohol intake within the UK Dietary Cohort Consortium. However, because of an insufficient number of participants in the heavier categories, a modest increased risk in those consuming $\geqslant 30 \mathrm{~g}$ per day cannot be excluded. Excessive alcohol intake has been causally related to numerous medical conditions (Rehm et al, 2003). Drinking-related morbidity and mortality constitute a large 
burden of diseases in Europe and worldwide (Ezzati et al, 2004; Rehm et al, 2006). In the United Kingdom, there was a substantial increase in both hospital admissions and deaths specifically related to alcohol misuse between 1991 and 2007, costing over $£ 2.7$ billion to the National Health Service annually (Rachel et al, 2009). The risks of alcohol intake should therefore be carefully considered in any decisions about alcohol drinking.

\section{ACKNOWLEDGEMENTS}

The UK Dietary Cohort Consortium at the MRC Centre for Nutritional Epidemiology in Cancer Prevention and Survival is funded by the Medical Research Council. The cohort studies included in this consortium received funding from the British Heart Foundation; Cancer Research UK; the Department of Health, UK; the Food Standards Agency, UK; the Medical Research Council, UK; the Stroke Association, UK; and the World Cancer Research Fund.

\section{Conflict of interest}

The authors declare no conflict of interest.

Supplementary Information accompanies the paper on British Journal of Cancer website (http://www.nature.com/bjc)

\section{REFERENCES}

Akhter M, Kuriyama S, Nakaya N, Shimazu T, Ohmori K, Nishino Y, Tsubono Y, Fukao A, Tsuji I (2007) Alcohol consumption is associated with an increased risk of distal colon and rectal cancer in Japanese men: the Miyagi Cohort Study. Eur J Cancer 43: 383-390

Allen NE, Beral V, Casabonne D, Kan SW, Reeves GK, Brown A, Green J, on behalf of the Million Women Study Collaborators (2009) Moderate alcohol intake and cancer incidence in women. J Natl Cancer Inst 101: 296-305

Allen NE, Roddam AW, Allen DS, Fentiman IS, dos Santos Silva I, Peto J, Holly JMP, Key TJ (2005) A prospective study of serum insulin-like growth factor-I (IGF-I), IGF-II, IGF-binding protein-3 and breast cancer risk. Br J Cancer 92: $1283-1287$

Appleby PN, Thorogood M, Mann JI, Key TJ (1999) The Oxford Vegetarian Study: an overview. Am J Clin Nutr 70: 525S - 5531

Baan R, Straif K, Grosse Y, Secretan B, El GF, Bouvard V, Altieri A, Cogliano V (2007) Carcinogenicity of alcoholic beverages. Lancet Oncol 8: $292-293$

Bingham S, Luben R, Welch A, Low YL, Khaw KT, Wareham N, Day N (2008) Associations between dietary methods and biomarkers, and between fruits and vegetables and risk of ischaemic heart disease, in the EPIC Norfolk Cohort Study. Int J Epidemiol 37: 978-987

Bingham SA, Gill C, Welch A, Cassidy A, Runswick SA, Oakes S, Lubin R, Thurnham DI, Key TJ, Roe L, Khaw KT, Day NE (1997) Validation of dietary assessment methods in the UK arm of EPIC using weighed records, and 24-h urinary nitrogen and potassium and serum vitamin C and carotenoids as biomarkers. Int J Epidemiol 26: S137-S151

Bingham SA, Luben R, Welch A, Wareham N, Khaw KT, Day N (2003) Are imprecise methods obscuring a relation between fat and breast cancer? Lancet 362: $212-214$

Bingham SA, Welch AA, McTaggart A, Mulligan AA, Runswick SA, Luben R, Oakes S, Khaw KT, Wareham N, Day NE (2001) Nutritional methods in the European Prospective Investigation of Cancer in Norfolk. Public Health Nutr 4: 847-858

Boffetta P, Hashibe M (2006) Alcohol and cancer. Lancet Oncol 7: 149-156 Bongaerts BW, van den Brandt PA, Goldbohm RA, de Goeij AF, Weijenberg MP (2008) Alcohol consumption, type of alcoholic beverage and risk of colorectal cancer at specific subsites. Int J Cancer 123: $2411-2417$

Brunner E, Stallone D, Juneja M, Bingham S, Marmot M (2001) Dietary assessment in Whitehall II: comparison of $7 \mathrm{~d}$ diet diary and foodfrequency questionnaire and validity against biomarkers. Br J Nutr 86: $405-414$

Bufill JA (1990) Colorectal cancer: evidence for distinct genetic categories based on proximal or distal tumor location. Ann Intern Med 113: $779-788$

Cade JE, Burley VJ, Greenwood DC (2004) The UK Women's Cohort Study: comparison of vegetarians, fish-eaters and meat-eaters. Public Health Nutr 7: $871-878$

Cade JE, Frear L, Greenwood DC (2006) Assessment of diet in young children with an emphasis on fruit and vegetable intake: using CADETChild and Diet Evaluation Tool. Public Health Nutr 9: 501-508

Carlin JB (2008) A new framework for managing and analyzing multiply imputed data in Stata. Stata J 8: 49-67

Chen K, Jiang Q, Ma X, Li Q, Yao K, Yu W, Zheng S (2005) Alcohol drinking and colorectal cancer: a population-based prospective cohort study in China. Eur J Epidemiol 20: 149-154
Cho E, Smith-Warner SA, Ritz J, van den Brandt PA, Colditz GA, Folsom AR, Freudenheim JL, Giovannucci E, Goldbohm RA, Graham S, Holmberg L, Kim DH, Malila N, Miller AB, Pietinen P, Rohan TE, Sellers TA, Speizer FE, Willett WC, Wolk A, Hunter DJ (2004) Alcohol intake and colorectal cancer: a pooled analysis of 8 cohort studies. Ann Intern Med 140: 603-613

Davey GK, Spencer EA, Appleby PN, Allen NE, Knox KH, Key TJ (2003) EPIC-Oxford: lifestyle characteristics and nutrient intakes in a cohort of 33883 meat-eaters and 31546 non meat-eaters in the UK. Public Health Nutr 6: $259-269$

Day N, Oakes S, Luben R, Khaw KT, Bingham S, Welch A, Wareham N (1999) EPIC-Norfolk: study design and characteristics of the cohort. European Prospective Investigation of Cancer. Br J Cancer 80(Suppl 1): $95-103$

Day NE, McKeown N, Wong MY, Welch A, Bingham S (2001) Epidemiological assessment of diet: a comparison of a 7-day diary with a food frequency questionnaire using urinary markers of nitrogen, potassium and sodium. Int J Epidemiol 30: 309-317

Department of Health (1999) Dietary Reference Values for Food Energy and Nutrients for the United Kingdom. HMSO Publications: London

Department of Health (2009) Health Profile of England 2008. Department of Health: London

Doll R, Peto R, Hall E, Wheatley K, Gray R (1994) Mortality in relation to consumption of alcohol: 13 years' observations on male British doctors. BMJ 309: $911-918$

Ely M, Hardy R, Longford NT, Wadsworth ME (1999) Gender differences in the relationship between alcohol consumption and drink problems are largely accounted for by body water. Alcohol Alcohol 34: 894-902

Ezzati M, Rodgers A, Lopez AD (2004) Mortality and burden of disease attributable to individual risk factors. In: Comparative Quantification of Health Risks. Global and Regional Burden of Disease Attributable to Selected Major Risk Factors. Volume 2 World Health Organization: Geneva

Ferrari P, Jenab M, Norat T, Moskal A, Slimani N, Olsen A, Tjonneland A, Overvad K, Jensen MK, Boutron-Ruault MC, Clavel-Chapelon F, Morois S, Rohrmann S, Linseisen J, Boeing H, Bergmann M, Kontopoulou D, Trichopoulou A, Kassapa C, Masala G, Krogh V, Vineis P, Panico S, Tumino R, van Gils CH, Peeters P, Bueno-de-Mesquita HB, Ocke MC, Skeie G, Lund E, Agudo A, Ardanaz E, Lopez DC, Sanchez MJ, Quiros JR, Amiano P, Berglund G, Manjer J, Palmqvist R, Van GB, Allen N, Key T, Bingham S, Mazuir M, Boffetta P, Kaaks R, Riboli E (2007) Lifetime and baseline alcohol intake and risk of colon and rectal cancers in the European prospective investigation into cancer and nutrition (EPIC). Int J Cancer 121: 2065-2072

Ferraroni M, Decarli A, Franceschi S, Vecchia CL, Enard L, Egri E, Arpinel M, Alvini S (1996) Validity and reproducibility of alcohol consumption in Italy. Int J Epidemiol 25: 775-782

Feunekes GIJ, van't Veer P, van Staveren WA, Kok FJ (1999) Alcohol intake assessment: the sober facts. Am J Epidemiol 150: 105-112

Giovannucci E, Rimm EB, Ascherio A, Stampfer MJ, Colditz GA, Willett WC (1995) Alcohol, low-methionine-low-folate diets, and risk of colon cancer in men. J Natl Cancer Inst 87: 265-273

Li FY, Lai MD (2009) Colorectal cancer, one entity or three. J Zhejiang Univ Sci B 10: $219-229$

Lim HJ, Park BJ (2008) Cohort study on the association between alcohol consumption and the risk of colorectal cancer in the Korean elderly. J Prev Med Public Health 41: 23-29 
Lindblom A (2001) Different mechanisms in the tumorigenesis of proximal and distal colon cancers. Curr Opin Oncol 13: 63-69

Marmot M, Brunner E (2005) Cohort profile: The Whitehall II study. Int J Epidemiol 34: 251-256

Mizoue T, Inoue M, Wakai K, Nagata C, Shimazu T, Tsuji I, Otani T, Tanaka K, Matsuo K, Tamakoshi A, Sasazuki S, Tsugane S, for the Research Group for the Development and Evaluation of Cancer Prevention Strategies in Japan (2008) Alcohol drinking and colorectal cancer in Japanese: a pooled analysis of results from five cohort studies. Am J Epidemiol 167: 1397-1406

Moskal A, Norat T, Ferrari P, Riboli E (2006) Alcohol intake and colorectal cancer risk: a dose-response meta-analysis of published cohort studies. Int J Cancer 120: 664-671

Prentice RL (2003) Dietary assessment and the reliability of nutritional epidemiology reports. Lancet 362: $182-183$

Rachel C, Jennifer M, Vasant H (eds) (2009) Health Survey for England 2008: Physical Activity and Fitness. Department of Health: London

Rehm J, Greenfield TK, Walsh G, Xie X, Robson L, Single E (1999) Assessment methods for alcohol consumption, prevalence of high risk drinking and harm: a sensitivity analysis. Int J Epidemiol 28: 219-224

Rehm J, Room R, Graham K, Monteiro M, Gmel G, Sempos CT (2003) The relationship of average volume of alcohol consumption and patterns of drinking to burden of disease: an overview. Addiction 98: 1209-1228

Rehm J, Taylor B, Patra J (2006) Volume of alcohol consumption, patterns of drinking and burden of disease in the European region 2002. Addiction 101: 1086-1095

Royston P (2005) Multiple imputation of missing values: update. Stata J 5: $188-201$
Schatzkin A, Kipnis V, Carroll RJ, Midthune D, Subar AF, Bingham S, Schoeller DA, Troiano RP, Freedman LS (2003) A comparison of a food frequency questionnaire with a $24-\mathrm{h}$ recall for use in an epidemiological cohort study: results from the biomarker-based Observing Protein and Energy Nutrition (OPEN) study. Int J Epidemiol 32: 1054-1062

Stang A, Kluttig A (2008) Etiologic insights from surface adjustment of colorectal carcinoma incidences: an analysis of the U.S. SEER data 2000 - 2004. Am J Gastroenterol 103: 2853-2861

Stewart B, Kleihues P (2003) World Cancer Report. IARC Press: Lyon

The National Health Service (2010) NHS choices: NHS advice on drinking limits. (http://www.drinking.nhs.uk/questions/recommended-levels/)

Toriola AT, Kurl S, Laukanen JA, Mazengo C, Kauhanen J (2008) Alcohol consumption and risk of colorectal cancer: the Findrink study. Eur $J$ Epidemiol 23: 395-401

Wadsworth M, Kuh D, Richards M, Hardy R (2006) Cohort profile: the 1946 National birth cohort (MRC National Survey of Health and Development). Int J Epidemiol 35: 49-54

WCRF/AICR (2007) Food, Nutrition, Physical Activity, and the Prevention of Cancer: a Global Perspective. AICR: Washington, DC

Welch AA, McTaggart A, Mulligan AA, Luben R, Walker N, Khaw KT, Day NE, Bingham SA (2001) DINER (Data Into Nutrients for Epidemiological Research) - a new data-entry program for nutritional analysis in the EPIC-Norfolk cohort and the 7-day diary method. Public Health Nutr 4: $1253-1265$

Westlake S, Cooper N (2008) Cancer incidence and mortality: trends in the United Kingdom and constituent countries, 1993 to 2004. Health Stat Quart 38: 33-46 